\title{
Factores predominantes que afectan el medio ambiente por el uso de la resina furanica en moldes para la fundicion de metales
}

\author{
Predominant factors that affect the environment due to the use of furanium \\ resin in molds for metal smelting
}

\author{
Carlos Del Valle Jurado ${ }^{1}$
}

Recibido: 09/08/2021 - Aprobado: 20/09/2021 - Publicado: 23/12/2021

\begin{abstract}
RESUMEN
La investigación parte del análisis de datos obtenidos de situaciones objetivas, generadas por los compuestos orgánicos volátiles en operaciones rutinarias de moldeo y fundición para así, poder determinar los factores predominantes que afectan la calidad ambiental por el uso de resina furánica en la preparación de moldes para la fabricación de piezas fundidas que se sustenta en el principio de cumplir con los objetivos propuestos en el reglamento de protección ambiental que regula el desarrollo de actividades en la industria manufacturera, así como analiza dichas actividades durante el proceso de producción en la fabricación de las piezas mencionadas, asumiendo y verificando los impactos ambientales que se generen en el área de influencia directa previamente determinada, proponiendo medidas preventivas que mitiguen y corrijan las posibles desviaciones en los procesos que alteren el equilibrio del medio físico, biológico y de relaciones socioeconómicas con las zonas de influencia. El desarrollo del trabajo se hizo tomando como referencia con muestras representativas de los procesos de fabricación de moldes y de la fundición del metal, en un área industrial clasificada y autorizada para tal fin, en la que se elaboran diversos tipos de piezas para la industria nacional. Se consideró normas legales nacionales y normas internacionales de manera general, así como de modo específico, aplicables a la generación de compuestos orgánicos volátiles para analizar los procesos productivos de una planta de fundición, se detallan los procesos, secuencia y actividades que se desarrollan en los procesos de fabricación.
\end{abstract}

Palabras claves: Arena recuperada para moldeo; pérdidas por ignición; contaminación del aire por compuestos orgánicos volátiles; emisiones; impacto ambiental.

\begin{abstract}
The research starts from the analysis of data obtained from objective situations, generated by volatile organic compounds in routine molding and casting operations in order to determine the predominant factors that affect environmental quality due to the use of furan resin in the preparation of molds for the manufacture of cast parts that is based on the principle of complying with the objectives proposed in the environmental protection regulation that regulates the development of activities in the manufacturing industry, as well as analyzes such activities during the production process in the manufacture of parts mentioned, assuming and verifying the environmental impacts that are generated in the previously determined area of direct influence, proposing preventive measures that mitigate and correct the possible deviations in the processes that alter the balance of the physical and biological environment and of socioeconomic relations with the areas of influence. The development of the work was done taking as a reference with representative samples of the processes of manufacture of molds and metal casting, in an industrial area classified and authorized for this purpose, in which various types of pieces are made for the national industry. National legal standards and international standards were considered in general, as well as specifically, applicable to the generation of volatile organic compounds to analyze the productive processes of a foundry plant, the processes, sequence and activities that are developed in the manufacturing processes.
\end{abstract}

Keywords: Recovered sand for molding; losses on ignition; air pollution by volatile organic compounds; emissions; environmental impact.

\footnotetext{
1 Universidad Nacional Mayor de San Marcos, Facultad de Ingeniería Geológica, Minera, Metalúrgica y Geográfica, EAP Ing. Geológica, Lima, Perú. E-mail: cdelvallej@unmsm.edu.pe-ORCID: https://orcid.org/0000-0002-3040-1396
} 


\section{INTRODUCCIÓN}

En la búsqueda e identificación de los efectos negativos al medioante que causan las actividades operativas de las fundiciones, se encontró que es debido a la existencia de los proceso térmicos y al uso de compuestos y aditivos químicos o aglutinantes orgánicos; en razón de ello, los problemas que se plantean con respecto a las fundiciones, son los relacionados con la emisión de gases, la eliminación de los residuos y la exposición de las personas a elementos químicamente contaminantes, que alteran la calidad ambiental.

El proceso de fabricación de las piezas de fundición genera residuos sólidos procedentes de las arenas usadas para la preparación de moldes y machos y es que frecuentemente, la arena de moldeo se humedece y se mezcla con resina furánica líquida, la cual, al realizarse el proceso de conformación por moldeo Universidad de Málaga, contribuye en la calidad del gas expelido, es decir, contribuye a la contaminación ambiental (Universidad de Málaga, 2005).

El problema de la generación de residuos en las fundiciones, es debido a los sólidos provenientes de las arenas de moldeo, de las escorias y polvos que salen de los hornos, así como los materiales que revisten los mismos; se enfrenta un gran reto de poder deshacerse de toda la arena usada por la necesidad de contar con recurso humano calificado y logística para todo el trabajo, ya que los residuos mencionados contienen aproximadamente 95\% de arena de sílice, siendo el resto, carbón mineral, arcilla, etc., además, es frecuente que la arena proveniente del desmoldeo de las piezas y de las zarandas vibratorias, se recicle para la fabricación de moldes nuevos, las que luego generan inherentemente polvo y desechos.

En el contexto descrito, el riesgo ambiental que supone el uso de la resina furánica que es utilizada en la preparación de moldes y machos para la fabricación de piezas fundidas; se debe, a que en promedio en el Perú los trabajadores encargados de la preparación de los moldes para fundición, habitualmente trabajan ocho hrs. por día y seis días a la semana; luego, la contaminación debido a la eliminación de los residuos se debe a que en dicha actividad, los trabajadores tienen exposición directa a la contaminación; pero, en el ámbito local se pudo detectar que las personas involucradas directamente en el trabajo habitual con el mencionado compuesto químico, conocen del peligro asociado, pero la necesidad de una retribución económica para cubrir los gastos básicos familiares dentro de una estructura social local precaria en el sentido económico, hace que la exposición y riesgo relacionados de manera directamente proporcional aumente el peligro de daño.

\section{MÉTODOS}

\subsection{Contaminación de aire por emisiones de gases (CO; NO2; SO2) y de otros Compuestos Orgánicos Volátiles (COV's)}

Sobre la base de la evaluación química de las arenas recuperadas o gastadas, se analizó el contenido de Compuestos Orgánicos Volátiles (COV's) y otros gases
(EPA, 2000), (Sánchez Montero \& Alcantara Leon, 2008). Cuando los moldes con aglutinantes orgánicos, son vertidos con aleaciones líquidas de metal a altas temperaturas $\left(1300{ }^{\circ} \mathrm{C}-1600{ }^{\circ} \mathrm{C}\right)$, una reacción térmica se produce y la descomposición de diversos gases contaminantes como $\mathrm{CO} ; \mathrm{NO}_{2} ; \mathrm{SO}_{2}$ y otras emisiones de sustancias peligrosas como los COV - compuestos orgánicos volátiles, entre ellos los HAP - hidrocarburos aromáticos policíclicos, además del Benceno, Tolueno, Etilbenceno y Xilenos BTEX (Cerón-Bretón et al., 2015), (ATSDR, 2016), de los cuales, una parte significativa se emite a la atmósfera durante el vaciado del molde y luego en el momento del enfriado, sin embargo, una parte de ellos se condensa en espacios intergranulares en arenas de moldeo las cuales son desechadas o recuperadas para un nuevo proceso de moldeo (Mastandrea et al., 2005).

Lo que da lugar a la mayor preocupación, porque contribuye significativamente a la contaminación ambiental, ennegrece el entorno de la fundición, paredes, suelos, ventanas y más. Los aglutinantes de furano han demostrado ser cancerígenos y se descomponen con emisión de gases tóxicos.

\subsection{Propiedades de la Resina Furánica y Catalizador}

Productos y agentes químicos habituales en el moldeo con resinas autofraguantes:

Resinas fenólicas: Formaldehído, fenol, hidróxido de sodio, hidróxido de potasio.

Resinas furánicas: Alcohol furfurílico, fenol, formaldehído, formol, úrea.

Endurecedores: Gamma-butirolactona, carbonato de propileno.

Catalizadores: Ácido paratoluensulfónico, ácido sulfúrico.

"Los agentes químicos más importantes desde el punto de vista toxicológico son, el formaldehído, alcohol furfurílico y fenol. La principal vía de entrada en el organismo para el formaldehído y el alcohol furfurílico es la vía inhalatoria, mucho menos importantes son la vía dérmica y los ojos, en el caso del fenol, las vías principales son la dérmica y los ojos, la inhalación es menos importante". BASEQUIM 018 Situaciones de Exposición a Agentes Químicos - Gobierno de España (Instituto Nacional de Seguridad, Salud y Bienestar en el trabajo, INSHT (2015).

Las arenas autofraguantes utilizan resina de furano a base de alcohol furfurílico y un catalizador ácido, tal y como se muestra en las Tablas 1 y 2 . Comparación de resultados de un tipo de resina furánica según especificación u hoja técnica y los valores reales encontrados.

\subsection{Pérdida de Peso por Ignición (L.O.I.)}

Se precisó de la metodología usada para poder determinar el contenido de material orgánico, que es el L.O.I., determinación de la pérdida de peso por ignición. 
Tabla 1. Propiedades resina furánica

\begin{tabular}{lcc}
\hline Parámetros & Especificación Proveedor & Valor Real \\
\hline$\%$ Alcohol Furfurilico & $50-65$ & No se determinó \\
$\%$ Sólidos $\left(135^{\circ} \mathrm{C}, 3.15-1 \mathrm{~h}\right)$ & No Reporta & 51 \\
$\%$ Humedad $(\mathrm{KF})$ & No Reporta & 17.45 \\
Índice de acidez (mg KOH/g muestra) & No Reporta & 1.1852 \\
$\mathrm{pH}\left(25^{\circ} \mathrm{C}\right)$ & $6.5-8.5$ & 6.85 \\
Formol Libre $(\%$ peso) & $<0.1$ & 0 \\
Viscosidad $\left(25^{\circ} \mathrm{C}, \mathrm{CP}\right)$ & $<100$ & 105 \\
Índice de Referencia $\left(20^{\circ} \mathrm{C}\right)$ & $1.4860-1.5100$ & 1.5064 \\
Cenizas $(\%$ Peso) & No Reporta & 0.58 \\
Nitrógeno $(\%$ Peso) & Libre de Nitrógeno & 0.12 \\
\hline
\end{tabular}

Tabla 2. Propiedades del catalizador

\begin{tabular}{ccc}
\hline Parámetros & Especificación Proveedor & Valor Real \\
\hline Ácido Xilen-Sulfónico (\% peso) & $83-91$ & 85.35 \\
Viscosidad $25^{\circ} \mathrm{C}$ & $260-300$ & 540 \\
Densidad $\left(25^{\circ} \mathrm{C}, \mathrm{g} / \mathrm{cm}^{3}\right)$ & $1.230-1.265$ & 1.2539 \\
Índice de acidez (mg KOH $/ \mathrm{g}$ muestra) & No Reporta & 268.40 \\
$\%$ Humedad (KF) & No Reporta & 4.90 \\
Ácido Sulfúrico Libre $(\%$ peso) & $<0.1$ & 3.37 \\
$\mathrm{pH} \mathrm{a} 25^{\circ} \mathrm{C}$ & $<0.1$ & Cumple \\
\hline
\end{tabular}

"Las pérdidas por ignición se refieren a la diferencia de peso antes y después de la ignición de una muestra de arena. Esta prueba es empleada para determinar la presencia de materiales orgánicos o de otro tipo que puedan formar gases en la mezcla de arena o en sus constituyentes, incluyendo arenas nuevas y recuperadas con mezcla de resina y catalizador. La medición de L.O.I. indica la cantidad de combustibles en la arena quemada, por lo tanto, las mediciones L.O.I. pueden proporcionar información esencial acerca de la calidad general del sistema de arena para fundición, la presencia de materiales orgánicos en arenas de fundición es problemática porque estos materiales pueden volatilizarse a temperaturas mucho más bajas que las requeridas para el proceso de fundición".

Al analizar el uso del método, se pudo determinar que el L.O.I. proporciona datos e información de manera rápida y eficiente para poder hallar el contenido de materia orgánica. La precision y eficacia del método, ayuda significativamente en comparación con otros métodos químicos más sofisticados.

Materiales y Equipos según Handbook de la AFS (Sociedad Americana de Fundición)

A. Procedimiento: AFS 5100-00-S (5-1)

B. Loss On Ignition, L.O.I.

La pérdida de peso de una muestra a $982^{\circ} \mathrm{C}$ es la pérdida de peso debido a la volatilización de sustancias orgánicas, el aumento de peso debido a las reacciones de oxidación (específicamente cromita, olivino y magnetita)
C. Equipos y materiales requeridos

- Balanza de $0.001 \mathrm{~g}$ de sensibilidad

- Dispositivo de secado con flujo de aire $\left(107^{\circ} \mathrm{C}+-\right.$ $\left.3^{\circ} \mathrm{C}\right)$

- Horno de mufla $\left(982+-6^{\circ} \mathrm{C}\right)$

- Crisoles refractarios

- Tenazas

- Desecador

- Ladrillos refractarios para apoyar crisoles calientes

D. Calculo de Resultados

$$
\% \mathrm{LOI}=\left[\frac{\mathrm{B}+\mathrm{C}}{\mathrm{B}-\mathrm{A}}\right] \times 100
$$

Actualmente, casi todas las plantas de fundición que utilizan arenas de moldeo con resina furánica, reutilizan el material recuperado en cantidades de 50 a $99 \%$. La pérdida de material recuperado en la ignición (L.O.I.) constituye una medida de sustancias orgánicas. Cuando el metal líquido es vertido en los moldes de arenas preparados con adiciones de regeneración de arena, aumentan las emisiones de gases $\left(\mathrm{CO} ; \mathrm{NO}_{2} ; \mathrm{SO}_{2}\right)$ y otros, como los Compuestos Orgánicos Volátiles (COV's) (EPA, 2000), las que hacen referencia a las sustancias o compuestos que contienen los productos 
químicos que se convierten fácilmente en vapores o gases, entre ellos los del grupo BTEX (benceno, tolueno, etilbenceno y xilenos) y HAPs (Hidrocarburos Aromáticos Policiclicos) (Cerón-Bretón et al., 2015), (ATSDR, 2016).

Los resultados de las investigaciones de descomposición térmica de arenas de moldeo preparadas con resinas furánicas, en las que la arena nueva es la base, se muestra en la Tablas 3 y 4; 1 - 1.5\% de Resina Furánica, $20-40 \%$ Catalizador

Tabla 3. Porcentaje (\%) L.O.I. arena para moldeo

\begin{tabular}{|c|c|c|c|}
\hline \multirow{2}{*}{ Meses de Muestreo } & \multicolumn{3}{|c|}{ \% L.O.I. } \\
\hline & $100 \mathrm{AN}$ & 25AN/75AR & 100AR \\
\hline Marzo & 1.38 & 4.98 & 5.34 \\
\hline Junio & 1.65 & 5.52 & 5.78 \\
\hline Setiembre & 1.43 & 5.21 & 5.48 \\
\hline Diciembre & 1.29 & 4.85 & 5.25 \\
\hline
\end{tabular}

Fuente: Elaboración Propia

Tabla 4. Ph de Arena de Moldeo

\begin{tabular}{cccc}
\hline Meses de Muestreo & \multicolumn{3}{c}{ Ph } \\
& 100AN & 25AN/75AR & 100AR \\
\hline Marzo & 4.35 & 3.81 & 2.51 \\
Junio & 4.85 & 3.21 & 2.35 \\
Setiembre & 4.63 & 3.52 & 2.41 \\
Diciembre & 4.49 & 3.95 & 2.71 \\
\hline
\end{tabular}

Fuente: Elaboración Propia

\subsection{Monitoreo ambiental}

Se realizó con el objetivo de obtener información sobre la presencia y concentración de elementos nocivos en el ambiente.

\subsubsection{Estándares Ambientales de Calidad del} Aire (ECA-Aire)

D.S. $N^{\circ}$ 003-2017-MINAM - Aprueba ECA para aire y disposiciones complementarias, aprobado el 07 de Junio del 2017, deroga el D.S. No 074-2001-PCM, el D.S. N ${ }^{o}$
069-2003-PCM, el D.S. No 003-2008-MINAM y el D.S. No006-2013-MINAM (Defensoria del Pueblo, 2017), (MINAM, 2015).

\subsubsection{Límites Máximos Permisibles (LMP)}

- $\quad$ R.M. No 315-96-EM/VMM, "Niveles Máximos Permisibles de Elementos y Compuestos presentes en Emisiones Gaseosas provenientes de las Unidades Minero - Metalúrgicas".

- Reglamento de valores límite permisibles para agentes químicos en el Ambiente de Trabajo.

- Environmental, Health, and Safety Guidelines, Foundries- World Bank Group, Abril 2007.

Límites máximos en la República Bolivariana de Venezuela: D.P. 638 - Normas de Calidad del aire y Control de la Contaminación Atmosférica (Monroy Gonzalez, 2008).

\subsection{Monitoreo de la Calidad del Aire}

A. Partículas Menores a 10 micras (PM10)

La tabla 5 muestra a los resultados de la concentración de PM10 en las dos estaciones de monitoreo de calidad de aire.

\section{B. Dióxido de Azufre ( $\left.\mathrm{SO}_{2}\right)$}

La tabla 6 muestra a los resultados de la concentración de Dióxido de Azufre $\left(\mathrm{SO}_{2}\right)$ en las dos estaciones de monitoreo de calidad de aire.

\section{Dioxido de Nitrógeno $\left(\mathrm{NO}_{2}\right)$}

La tabla 7 muestra los resultados de la concentración de Dióxido de Nitrógeno $\left(\mathrm{NO}_{2}\right)$ en las dos estaciones de monitoreo de calidad de aire.

\section{Monoxido de Carbono (CO)}

La tabla 8 muestra los resultados de la concentración de "monóxido de carbono" (CO) en las estaciones instaladas para el monitoreo de calidad del aire. La tabla 9 muestra las emisiones de los hornos.

\section{E. Hidrocarburos aromáticos policíclicos (HAPs)}

La identificación de los HAPs se realizó mediante el sistema compuesto por el cromatógrafo de gases Trace GC

Tabla 5. Concentración de Partículas PM10

\begin{tabular}{ccccc}
\hline Estación de Monitoreo & Fecha de Muestreo & $\begin{array}{c}\text { Tiempo de Muestreo } \\
(\mathrm{min})\end{array}$ & $\begin{array}{c}\text { Concentración } \\
\left(\boldsymbol{\mu} \mathrm{m} / \mathrm{m}^{3}\right)\end{array}$ & $\begin{array}{c}\text { Estándar de Calidad Ambiental } \\
\text { para Aire }(\mathbf{1})\end{array}$ \\
\hline EM - 1 & Del 08 al 09 Enero del 2018 & 1440 & 61,4 & $100\left(\mu \mathrm{m} / \mathrm{m}^{3}\right)$ \\
EM - 2 & & & 135,5 & \\
\hline
\end{tabular}

(1) D.S. No 003-2017-MINAM

Tabla 6. Concentración de Dióxido de Azufre" $\left(\mathrm{SO}_{2}\right)$

\begin{tabular}{cccc}
\hline Estación de Monitoreo & Fecha de Muestreo & $\begin{array}{c}\text { Tiempo de Muestreo } \\
(\mathrm{min})\end{array}$ & $\begin{array}{c}\text { Concentración } \\
\left(\boldsymbol{\mu} / \mathrm{m}^{3}\right)\end{array}$ \\
\hline EM - 1 & Del 08 al 09 Enero del 2018 & 1440 & $<12,15$ \\
EM - 2 & & & $<12,15$ \\
\hline
\end{tabular}


Tabla 7. Concentración de Dióxido de Nitrógeno" $\left(\mathrm{NO}_{2}\right)$

\begin{tabular}{ccccc}
\hline Estación de Monitoreo & Fecha de Muestreo & $\begin{array}{c}\text { Tiempo de Muestreo } \\
(\mathrm{min})\end{array}$ & $\begin{array}{c}\text { Concentración } \\
\left(\mu \mathrm{m} / \mathrm{m}^{3}\right)\end{array}$ & $\begin{array}{c}\text { Estándar de Calidad Ambiental } \\
\text { para Aire }(1)\end{array}$ \\
\hline EM - 1 & Del 08 al 09 Enero del 2018 & 60 & $<8,75$ & $200\left(\mu \mathrm{m} / \mathrm{m}^{3}\right)$ \\
\hline
\end{tabular}

(1) D.S. No 003-2017-MINAM

Tabla 8.. Concentración de Monóxido de Carbono" (CO)

\begin{tabular}{ccccc}
\hline $\begin{array}{c}\text { Estación de } \\
\text { Monitoreo }\end{array}$ & Fecha de Muestreo & $\begin{array}{c}\text { Tiempo de Muestreo } \\
(\mathbf{m i n})\end{array}$ & $\begin{array}{c}\text { Concentración } \\
\left(\boldsymbol{\mu m} / \mathrm{m}^{3}\right)\end{array}$ & $\begin{array}{c}\text { Estándar de Calidad Ambiental } \\
\text { para Aire (1) }\end{array}$ \\
\hline EM -1 & Del 08 al 09 Enero del 2018 & 480 & $<652$ & $10000\left(\mu \mathrm{m} / \mathrm{m}^{3}\right)$ \\
EM - 2 & & & 660 & \\
\hline
\end{tabular}

(1) D.S. No 003-2017-MINAM

Tabla 9. Emisiones de los Hornos

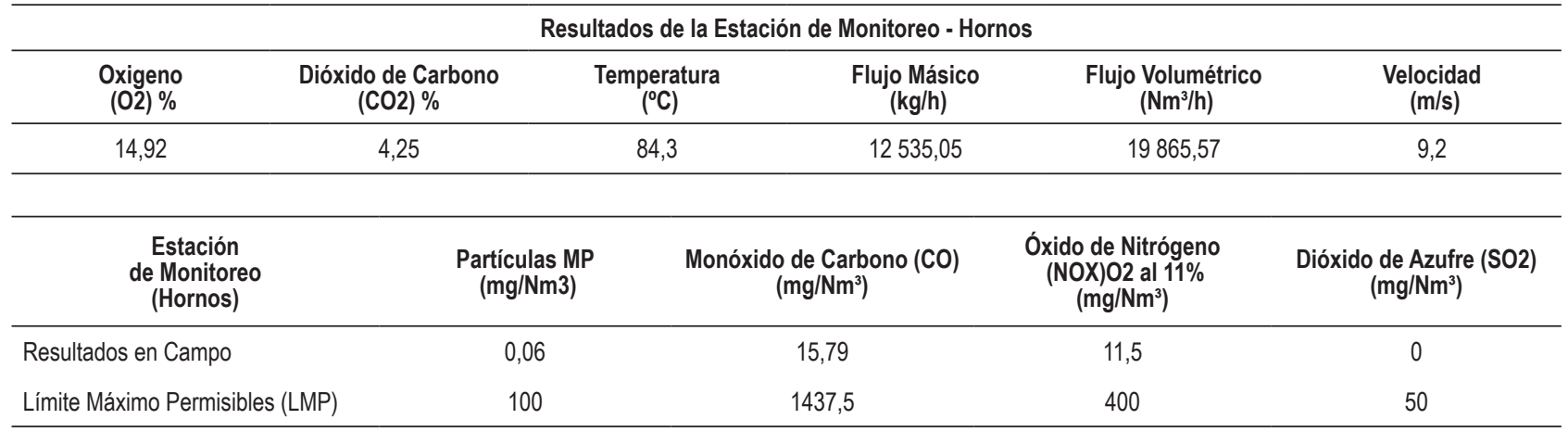

(1) Fuente: R.M. No 315-96-EM/VMM.

(2) Límites establecidos en la Republica Bolivariana de Venezuela: D.P. № 638 - Normas de calidad del aire y control de la contaminación atmosférica del 26/04/’95. Condiciones de Conversión de ppm a ug/m3: $P=1013.25$ mbar, $\mathrm{T}=25^{\circ} \mathrm{C}$. (Monroy Gonzalez, 2008).

(3) Environmental, Health, and Safety Guidelines Foundries World Bank Group Abril 2007. Fusión de metales ferrosos. Nivel máximo de emisiones para las mejores tecnologías disponibles (BAT) y para hornos de cubilote sin coque.

Ultra, equipado con la columna cromatográfica capilar RTX 5MS (Restek) de $30 \mathrm{~mm}$., de diámetro interno $0,25 \mathrm{~mm}$. El análisis de los compuestos del grupo HAPs se realizó según la norma ISO 11338-2: 2003, esta norma específica los procedimientos para la preparación de muestras, limpieza y análisis para la determinación de Hidrocarburos Aromáticos Policíclicos (HAP's) en fase gaseosa y de partículas en gases de chimenea y gases residuales (Mastandrea et al., 2005), (INSST, 2007). Los métodos analíticos son capaces de detectar concentraciones de HAP's de submicrogramos por metro cúbico de muestra, según el tipo de HAP's y el volumen de gases de combustión muestreados (ver tablas 10 y 11$)$.

Tabla 10. Metodología de Análisis de COV's

\begin{tabular}{cc}
\hline Parámetro & Referencia del Método \\
\hline Benzeno & \\
Etilbenzeno & Cromatografía de Gases \\
Tolueno & ISO 11338-2: 2003 \\
Xileno & \\
\hline
\end{tabular}

Tabla 11. Emisiones de Gases COV's Concentraciónes de BTEX's formados durante la descomposición térmica de arena de moldeo

\begin{tabular}{ccccc}
\hline \multirow{2}{*}{$\mathbf{N}^{\circ}$ CAS } & Agente Químico & \multicolumn{3}{c}{$\left(\boldsymbol{\mu g} / \mathrm{m}^{3}\right)$} \\
& & 100AN & 25AN/75AR & 100AR \\
\hline $71-43-2$ & Benzeno & 1.132 & 14.245 & 28.532 \\
$108-88-3$ & Tolueno & 0.845 & 2.542 & 4.485 \\
$100-41-4$ & Etilbenzeno & 0.253 & 0.425 & 0.652 \\
$1330-20-7$ & Xilenos, mezcla isómeros & 0.114 & 0.194 & 0.409 \\
$95-47-6$ & o-xileno & 0.025 & 0.045 & 0.075 \\
$108-38-3$ & m-xileno & 0.055 & 0.064 & 0.142 \\
$106-42-3$ & p-xileno & 0.034 & 0.085 & 0.192 \\
\hline
\end{tabular}

\section{F. Contaminación por exposición en ambienta laboral}

El desarrollo de actividades industriales generan riesgos para la salud en los diferentes ambientes de trabajo, es por ello la necesidad de identificar y medir la presencia de éstos y otros factores asociados a la diversas actividades para poder diagnosticarlas, corregirlas y prevenirlas con decisiones 
oportunas y efectivas. "En los procesos productivos existen múltiples contaminantes del ambiente laboral que actúan como factores de riesgo y que pueden incidir negativamente en la salud de los individuos que entran en contacto, entre estos factores, revisten especial importancia los agentes de origen químico, físico y biológico, de manera general, se definióla exposición, el contacto en el tiempo y el espacio entre una persona y uno o más agentes biológicos, químicos o físicos. El concepto de exposición depende de una serie de parámetros a considerar, por lo tanto, al momento de evaluar la exposición, se deben considerar estos aspectos. En términos de salud ocupacional, se define exposición como el acto o condición de estar, por razones de trabajo, en contacto dérmico, por inhalación o ingestión, con uno o una mezcla de estos agentes contaminantes, en un lugar y durante un período de tiempo determinado. La evaluación de la exposición en una determinado grupo de trabajadores, corresponde a la medición de la intensidad y/o duración y frecuencia del contacto del individuo con un agente nocivo específico, se utiliza para caracterizar el riesgo de un individuo o grupo de individuos expuestos.
La evaluación de la exposición en diferentes ambientes laboral implica identificar y evaluar los agentes que pueden entrar en contacto con los trabajadores y analizar diferentes aspectos del proceso que determina el contacto".

"Media Ponderada en el Tiempo (TWA).- Es la concentración media del agente químico en la zona de respiración del trabajador medida, o calculada de forma ponderada con respecto al tiempo, para la jornada estándar de 8 horas diarias".

A. "Exposición de corta duración: (STEL).- Es la concentración media del agente químico en la zona de respiración del trabajador, medida o calculada para cualquier período de 15 minutos a lo largo de la jornada laboral, excepto para aquellos agentes químicos para los que se especifique un período de referencia inferior, en la lista de Valores Límite". (Reglamento sobre valores limite permisibles para agentes químicos en el ambiente de trabajo; D.S. N N $^{\mathrm{o}}$ 015-2005-SA) (ver tablas $12,13,14$ y 15 ).

Tabla 12. "Reglamento sobre valores límite permisibles para agentes químicos en el ambiente de trabajo"

\begin{tabular}{|c|c|c|c|c|c|c|c|}
\hline \multicolumn{8}{|c|}{ "Valores Límite Permisibles para Agentes Quimicos en el Ambiente de Trabajo" } \\
\hline \multirow{3}{*}{$\mathrm{N}^{\circ} \mathrm{CAS}$} & \multirow{3}{*}{ Agente Químico } & \multicolumn{4}{|c|}{ "Limites Adoptados" } & \multirow{3}{*}{$\begin{array}{l}\text { Peso Molecular } \\
\text { (gramos) }\end{array}$} & \multirow{3}{*}{ Notawas } \\
\hline & & \multicolumn{2}{|c|}{ TWA } & \multicolumn{2}{|c|}{ STEL } & & \\
\hline & & ppm & $\mathrm{mg} / \mathrm{m}^{3}$ & ppm & $\mathrm{mg} / \mathrm{m}^{3}$ & & \\
\hline $100-41-4$ & Etilbenceno & 100 & 434 & 125 & 543 & 106,16 & Vía dérmica VLB \\
\hline $108-88-3$ & Tolueno & 50 & 188 & & & 92,13 & Vía dérmica VLB \\
\hline $1330-20-7$ & Xileno, mezcla isómeros & 100 & 434 & 150 & 651 & 106,16 & Vía dérmica VLB \\
\hline $95-47-6$ & o-xileno & 100 & 434 & 150 & 651 & 106,16 & Vía dérmica VLB \\
\hline $108-38-3$ & m-xileno & 100 & 434 & 150 & 651 & 106,16 & Vía dérmica VLB \\
\hline $106-42-3$ & p-xileno & 100 & 434 & 150 & 651 & 106,16 & Vía dérmica VLB \\
\hline
\end{tabular}

Fuente: D.S. N $015-2005-S A$

Tabla 13. Valores Límite Permisibles

Valores Límite Permisibles para Agentes Quimicos Cancerigenos en el Ambiente de Trabajo

\begin{tabular}{|c|c|c|c|c|c|}
\hline \multicolumn{6}{|c|}{ Valores Límite Permisibles para Agentes Quimicos Cancerigenos en el Ambiente de Trabajo } \\
\hline \multirow{3}{*}{ CAS } & \multirow{3}{*}{ Categoría } & \multirow{3}{*}{ Agente Químico } & \multirow{2}{*}{\multicolumn{2}{|c|}{$\begin{array}{l}\text { Límites Adoptados } \\
\text { TWA }\end{array}$}} & \multirow{3}{*}{ Notas } \\
\hline & & & & & \\
\hline & & & ppm & $\mathrm{mg} / \mathrm{m}^{3}$ & \\
\hline $65996-93-2$ & A1 & $\begin{array}{l}\text { Alquitrán de hulla, elevada temp. } \\
\text { Brea comp. Volátil como soluble en Benceno }\end{array}$ & & 0.2 & Vía dérmica, VLB \\
\hline $71-43-2$ & $\mathrm{~A} 1$ & Benceno & 0.5 & 1.6 & Vía dérmica, VLB \\
\hline
\end{tabular}

Fuente: D.S. N ${ }^{0}$ 015-2005-SA (ATSDR, 2016).

Tabla 14. Listado de Agentes Quimicos Cancerigenos cuyo contacto debe evitarse*

\begin{tabular}{ccc}
\hline CAS & Categoría & Agente Químico \\
\hline $50-32--8$ & A2 & Benzopireno \\
$205-99-2$ & A2 & Benzofluoranteno \\
$569-55-3$ & A2 & Benzoantraceno \\
$97-87-5$ & A1 & Bencidina \\
& & Dimetilaminoazobenzeno \\
\hline
\end{tabular}

Fuente: D.S. No 039-93-PCM "Reglamento de Prevención y Control del Cancer Profesional"

*"Uso en estado puro o mezclado con otras sustancias cancerigenas o no". 
Tabla 15. Emisión de gases COV's concentración de HAP's formados durante la descomposición térmica arena de moldeo

\begin{tabular}{|c|c|c|c|c|}
\hline \multirow{2}{*}{$\mathrm{N}^{0}$ CAS } & \multirow{2}{*}{ Agente Químico } & \multicolumn{3}{|c|}{$\left(\mathrm{mg} / \mathrm{m}^{3}\right)$} \\
\hline & & $100 \mathrm{AN}$ & 25AN/75AR & 100AR \\
\hline $91-20-3$ & Naptaleno & 28.42 & 112.55 & 140.55 \\
\hline $83-32-9$ & Acenafteno & * & * & * \\
\hline $208-96-3$ & Acenaftileno & 1.18 & 3.47 & 5.12 \\
\hline $86-73-7$ & Fluoreno & 3.53 & 3.31 & 7.35 \\
\hline $85-01-8$ & Fenantreno & 0.51 & 1.03 & 2.35 \\
\hline $120-12-7$ & Antraceno & 3.85 & 4.55 & 8.23 \\
\hline $206-12-7$ & Fluoranteno & 5.55 & 8.37 & 9.08 \\
\hline $129-00-0$ & Pireno & 3.52 & 3.86 & 7.35 \\
\hline $56-55-3$ & Benzo antraceno & 0.81 & 2.98 & 3.93 \\
\hline 218-01-19 & Criseno & 0.10 & 0.18 & 0.25 \\
\hline $205-99-2$ & Benzo fluoranteno & 3.70 & 5.83 & 6.92 \\
\hline $207-08-9$ & Benzo fluoranteno & 1.23 & 1.35 & 1.96 \\
\hline $50-32-8$ & Benzo pireno & 0.95 & 3.47 & 4.09 \\
\hline $53-70-3$ & Dibenzo antraceno & 0.22 & * & * \\
\hline $191-24-2$ & Benzo perileno & 0.39 & 0.61 & 0.85 \\
\hline $193-39-5$ & Indeno pireno & * & * & * \\
\hline $132-64-9$ & Dibenzofurano & 0.05 & 0.05 & 0.07 \\
\hline $90-12-0$ & 1-Metilnaftaleno & 0.18 & 0.35 & 0.41 \\
\hline $91-57-6$ & 2-Metilnaftaleno & 0.25 & 0.47 & 0.47 \\
\hline $92-52-4$ & Bifenilo & 0.06 & 0.09 & 0.10 \\
\hline
\end{tabular}

\section{RESULTADOS}

La investigación indica que las adiciones de arena recuperada a las arenas de moldeo, tienen una influencia significativamente negativa sobre el medio ambiente y la salud de los trabajadores, principalmente se trata de compuestos orgánicos volátiles (COV's) y sustancias químicas, cuyas cantidades aumentan junto con el aumento de arena recuperada (EPA, 2000), (Sánchez Montero \& Alcantara Leon, 2008).

A mayor cantidad de arena recuperada en la arena de moldeo, aumentan las concentraciones de los compuestos del grupo BTEX's (Cerón-Bretón et al., 2015), durante el vertido del metal al molde se puede apreciar que estás aumentan significativamente (casi tres veces). La concentración de benceno (ATSDR, 2016), (componente principal de este grupo de propiedades cancerígenas aumenta más que los otros compuestos. Todas estas concentraciones son proporcionales a los valores de L.O.I.

Adiciones de arena recuperada en la arena de moldeo provocan el aumento del valor de L.O.I. y en consecuencia, la emisión de mayores volúmenes de gases.

Con respecto al grupo HAP's para la arena de moldeo que contiene el 75\% y $99 \%$ de arena recuperada, se observa también un considerado aumento con respecto a la arena nueva de sustancias como; Benzo(a)antraceno; Benzo(b) fluoranteno y Benzo(a) pireno; según el listado de agentes químicos cancerígenos del decreto Supremo No 015-2015SA (Reglamento sobre Valores Limite Permisibles para Agentes Químicos en el Ambiente de Trabajo), el contacto con estas sustancias en estado puro o mezclado deben evitarse.

Casi el 50\% de las sustancias del grupo de los HAP's emitidas en el proceso de vertido del metal líquido al molde constituía naftaleno, que está prácticamente adsorbido en su totalidad sobre la espuma de poliuretano.

En líneas generales, las actividades generadas por la Fundición, inciden negativamente en la calidad del aire del área monitoreada, ya que existe concentraciones de compuestos organicos volátiles (COV) que se encuentran por encima de los estándares de calidad ambiental tomados como referencia.

\section{CONCLUSIONES}

1. Las Investigaciones realizadas en condiciones normales, indicaron una influencia significativa de la cantidad de regeneración en la matriz de arena de moldeo sobre la emisión de sustancias del grupo HAPs y BTEXs, por lo que, cuando se aplique una adición de arena recuperada, se debe prestar especial atención al aumento significativo de la emisión de sustancias nocivas para la salud y el medio ambiente. 
2. Es importante y resulta práctico, utilizar el porcentaje de L.O.I. como indicador de contaminación en arenas recuperadas en el caso de arenas con aglutinantes orgánicos en especial resinas furánicas, ya que es un método rápido, fácil y económico. La similar dependencia de los grupos HAPs y BTEXs y la pérdida por ignición de la cantidad de arena recuperada de moldeo, proporcionan la base para la evaluación de la nocividad de estas arenas (Cerón et al).

3. La presente investigación debe utilizarse como herramienta para futuras evaluaciones ambientales en fundiciones que utilizan como base de moldeo resinas furánicas con altos contenidos de alcohol furfurílico $(>60 \%)$ y presencia de nitrógeno en su formulación, del mismo modo se debe tener tener en cuenta que la formulación del catalizador o endurecedor debería tener como contenido de ácido sulfúrico $\left(\mathrm{H}_{2} \mathrm{SO}_{4}\right)<0.1$ (\% en peso) o en el mejor de los casos cero.

4. Con legislaciones ambientales cada vez más estrictas, es necesario realizar investigaciones como la que se presenta, para apoyar de manera técnica en los procesos de operación sostenible de fabricación de piezas de metal. La escasa o nula información de datos experimentales para describir las características químicas en el comportamiento de compuestos tóxicos de estas arenas recuperadas ha hecho de esta investigación un valioso aporte para el control ambiental que mantenemos en la actualidad.

5. Es necesario investigar y monitorear con mayor detalle los Compuestos Organicos Volátiles (COV's) de otro tipo de resinas y catalizadores autofraguantes, para implementar proyectos de investigación que determinen las fuentes de contaminantes orgánicos, para disminuir los riesgos y evitar la biomagnificación y desarrollo de enfermedades por agentes contaminantes.

\section{REFERENCIAS}

ATSDR. (2016). Resumen de Salud Pública: Benceno. Agency For Toxic Substances And Disease Registry. https://www. atsdr.cdc.gov/es/phs/es_phs3.html

Cerón-Bretón, J. G., Cerón-Bretón, R. M., Kahl, J. D. W., RamírezLara, E., Guarnaccia, C., Aguilar-Ucán, C. A., MontalvoRomero, C., Anguebes-Franseschi, F., \& López-Chuken, U. (2015). Diurnal and seasonal variation of BTEX in the air of Monterrey, Mexico: preliminary study of sources and photochemical ozone pollution. Air Quality, Atmosphere and Health, 8(5), 469-482. https://doi.org/10.1007/s11869014-0296-1
Defensoria del Pueblo. (2017). La calidad del aire en Lima y su impacto enla salud y la vida de sus habitantes. Informe Defensorial, 116, 1-82. www.defensoria.gob.pe

EPA. (2000). Determinación de compuestos orgánicos volátiles (COVs) en el aire recolectado en recipientes especialmente preparados y analizado por cromatografía de gases / espectrometría de masas (GC/MS). In The Hostory of Drinking water Treatment. https://nepis.epa. gov/Exe/ZyNET.exe/P100YDPO.TXT?ZyActionD= ZyDocument\&Client $=$ EPA\&Index $=2016+$ Thru +2020 $\&$ Docs $=\&$ Query $=\&$ Time $=\&$ EndTime $=\&$ SearchMethod $=1 \&$ TocRestrict $=$ n $\&$ Toc $=\&$ TocEntry $=\& Q F i e l d=$ $\&$ QField Year $=\& Q$ FieldMonth $=\& Q$ FieldDay $=\&$ IntQ FieldOp $=0 \&$ ExtQ FieldOp $=0 \& X m l Q u e r y=$

INSHT. (2015). BASEQUIM SITUACIONES DE EXPOSICIÓN A AGENTES QUÍMICOS. https:// ww w.ins st.es/documents/94886/791398/ BASEQUIM_018_0.pdf/aba6e604-4f16-4674-834b$437 \mathrm{ff} 67425 \mathrm{~b} 6 \overline{\mathrm{t}}=16 \overline{0} 6302597424$

INSST. (2007). PROPIEDADES DE LOS HIDROCARBUROS AROMATICOS. 282-283. https://www.insst.es/doc uments/94886/162038/6.+Hidrocarburos+aromátic os+-+Hidrocarburos+aromáticos+halogenados+++Hidrocarburos+poliaromáticos++-+-Isocianatos+-+Cetonas\#: :text=Los hidrocarburos aromáticos son aquellos,los vértices de un hexágono.

Mastandrea, C., Chichizola, C., Ludueña, B., Sánchez, H., Álvarez, H., \& Gutiérrez, A. (2005). Hidrocarburos aromáticos policíclicos. Riesgos para la salud y marcadores biológicos. In Acta Bioquimica Clinica Latinoamericana (Vol. 39, Issue 1, pp. 27-36). https://www.redalyc.org/ pdf/535/53522191006.pdf

MINAM. (2015). Guía para la elaboración de estudios de Evaluación de Riesgos a la Salud y el Ambiente. Ministerio Del Ambiente, 160. https://www.minam.gob. pe/calidadambiental/wp-content/uploads/sites/22/2015/02/ GUIA-ERSA-ALTA.compressed.pdf

Monroy Gonzalez, E. C. (2008). Estudio de factibilidad de generación de dioxinas y furanos (PCDD y PCDF) en el proceso de reformacion catalitica de naftas en la industria petrolera y propuestas para minimizar su emision.

Sánchez Montero, J. M., \& Alcantara Leon, A. (2008). Compuestos orgánicos volátiles en el medio ambiente. Monografias de La Real Academia Nacional de Farmacia, 27. https://core. ac.uk/download/pdf/230313907.pdf

Universidad de Málaga. (2005). Introducción al conformado por moldeo. http://www.raquelserrano.com/wp-content/files/ procesos_Fundicion_0.pdf 